\title{
Clinical Outcomes of Argon Plasma Coagulation Therapy for Early Gastric Neoplasms
}

\author{
Kyu Young Kim, Seong Woo Jeon, Hea Min Yang, Yu Rim Lee, Eun Jeong Kang, Hyun Seok Lee and \\ Sung Kook Kim \\ Department of Internal Medicine, Kyungpook National University School of Medicine, Daegu, Korea
}

Background/Aims: Argon plasma coagulation (APC) has some merits in the treatment of gastric neoplasms including a shorter operative time and fewer complications compared with endoscopic mucosal resection or endoscopic submucosal dissection. However, there are few reports on the outcomes of gastric neoplasms treated using APC. The aim of this study was to evaluate APC in the treatment of early gastric neoplasms in terms of clinical efficacy, safety, and local recurrence.

Methods: We enrolled 28 patients who received APC therapy at the Kyungpook National University Hospital between May 2007 and April 2013. Clinical outcomes were analyzed.

Results: The median follow-up period was 24.8 months (range, 2 to 78). Among the 28 lesions treated using the APC procedure, tumor recurrence was encountered in seven lesions (25.0\%). Recurrence was found in 50\% (5/10) of single APC cases and $11 \%(2 / 18)$ of rescue APC cases. The mean time to recurrence was 16.1 months (range, 2 to 78 ). There were no serious APC-related complications such as perforation, bleeding, or infection.

Conclusions: APC therapy can be a useful treatment with a favorable safety profile for patients with early gastric neoplasms. However, further studies are necessary to determine the long-term prognosis of patients undergoing this treatment.

Key Words: Early gastric cancer; Recurrence; Endoscopic treatment; Argon plasma coagulation

\section{INTRODUCTION}

Endoscopic mucosal resection (EMR) and endoscopic submucosal dissection (ESD) are important treatments for early gastric cancer (EGC) or gastric adenoma. ${ }^{1-3}$ However, these endoscopic resections, particularly ESD, require specialist skills and a long operation time. In addition, endoscopic resection cannot be performed in cases with a highrisk of bleeding or perforation or in cases of non-lifting after submucosal injection.

Argon plasma coagulation (APC) is a noncontact tissue coagulation technique that transfers a high-frequency electric current through ionized argon gas to the target tissue. ${ }^{4,5}$ An

Received: February 27, 2014 Revised: May 24, 2014

Accepted: June 14, 2014

Correspondence: Seong Woo Jeon

Gastric Cancer Center, Kyungpook National University Medical Center, Kyungpook National University School of Medicine, 807 Hoguk-ro, Buk-gu, Daegu 702-210, Korea

Tel: +82-53-200-2602, Fax: +82-53-200-2028, E-mail: sw-jeon@daum.net

(c) This is an Open Access article distributed under the terms of the Creative Commons Attribution Non-Commercial License (http://creativecommons.org/ licenses/by-nc/3.0) which permits unrestricted non-commercial use, distribution, and reproduction in any medium, provided the original work is properly cited.
APC applicator for endoscopic use was developed by Grund et al. ${ }^{4}$ in 1994. APC has been used mainly for hemorrhagic lesions and bleeding sites in the gastrointestinal tract. ${ }^{6,7}$ In EGC, APC has been used to treat patients who cannot undergo endoscopic resection or surgery. APC has several merits including a short operative time, ease of use, lack of serious complications, and operator independence. However, APC has been used in only a few patients because of the difficulty in predicting the depth of invasion and the inability to perform pathological evaluations, which are possible with complete resection. ${ }^{8-10}$

There have been few reports on the outcomes of patients who receive APC therapy for gastric neoplasms. Thus, the aim of this study was to determine the clinical outcomes of APC in patients with EGC or gastric adenoma.

\section{MATERIALS AND METHODS}

\section{Patients}

This study enrolled 32 patients (26 men and six women) 
who received singleor rescue APC (after ESD or EMR) for the treatment of EGC or gastric adenoma in our hospital between May 2007 and April 2013. Single APC treatment was selected instead of surgical or endoscopic resection for the following reasons: (1) the patient had a high surgical risk or refused surgery; (2) the lesion carried a high risk of complications or technical difficulties for endoscopic resection; or (3) the patient refused endoscopic resection owing to its high cost or the long duration of hospitalization. Rescue APC treatment was selected in cases with endoscopically uncertain or unclear margins. We observed well-differentiated EGCs in seven cases, moderately differentiated EGCs in two cases, poorly differentiated EGCs in five cases, and adenomas in 18 cases. Of these, three cases were excluded because of loss to follow-up after 3 months and one case was excluded for undergoing surgery for metachronous recurrence after 3 months. With the exception of one case, all excluded cases were adenomas. We obtained information on the patients who were lost to follow-up via telephone call with verbal consent. A total of 28 cases were retrospectively analyzed. This study was approved by the Institutional Review Board of the Kyungpook National University Medical Center.

\section{APC procedure}

All procedures were performed under conscious sedation. Cardiorespiratory functions were continually monitored throughout the procedure. The APC equipment included an argon gas source and a high-frequency generator (VIO300 D; Erbe Elektromedizin, Tübingen, Germany). The argon gas flow rate was $1.5 \mathrm{~L} / \mathrm{min}$ and the electrical current was set at $50 \mathrm{~W}$.

\section{Definitions}

Macroscopic EGC and adenoma types were classified according to Japanese Gastric Cancer Association criteria. ${ }^{11}$ The degree of differentiation was classified according to World Health Organization recommendations. Local recurrence was defined as recurrent cancer or adenoma at the resection site. Tumors detected at sites other than the primary resection site during follow-up endoscopy were regarded as metachronous recurrences. Significant bleeding was defined as bleeding with clinical symptoms or bleeding requiring transfusion.

\section{Follow-up schedule}

All patients were examined by conventional endoscopy at 3, 6, and 12 months after APC therapy. Tumor recurrence was confirmed histologically using biopsy specimens obtained from the initially treated lesions.

\section{RESULTS}

A flowchart of the enrolled patients is shown in Fig. 1. The median follow-up period (from the date of initial treatment to the date of final endoscopic examination or recurrence) was 24.8 months (range, 2 to 78 ). Table 1 summarizes the baseline characteristics of the 28 patients. Eleven patients had underlying diseases, including seven patients with cardiovascular disease (five with poorly controlled hypertension and two with ischemic heart disease). In addition, six patients had diabetes

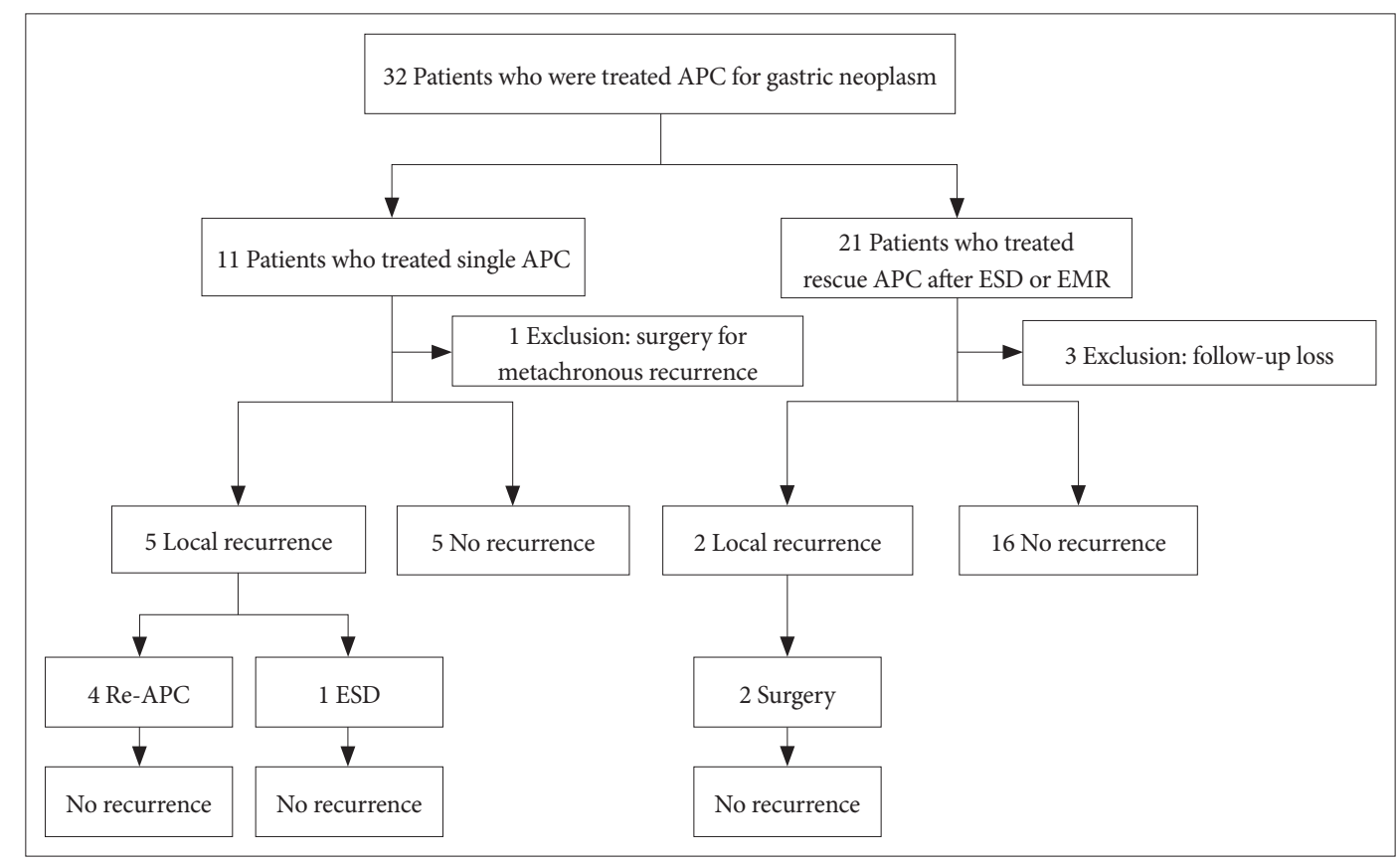

Fig. 1. Flowchart of inclusion criteria used in this study. The clinical outcomes of 28 patients with early gastric neoplasms were analyzed after argon plasma coagulation (APC). ESD, endoscopic submucosal dissection; EMR, endoscopic mucosal resection. 
mellitus and two patients had other malignancies (one with esophageal cancer and one with bladder cancer). Nine patients were older than 70 years.

Among the 28 lesions treated with an APC procedure, tumor recurrence was encountered in seven lesions (25.0\%) during the follow-up period. Recurrence was found in $50 \%$ $(5 / 10)$ of single APC cases and $11 \%(2 / 18)$ of rescue APC cases. Recurrence was located in the middle region in four patients, the lower region in one patient, and the upper region in two patients. The histological diagnosis of these lesions was EGC

Table 1. Characteristics of Patients Treated with Argon Plasma Coagulation $(n=28)$

\begin{tabular}{lc}
\hline \multicolumn{1}{c}{ Characteristic } & Value \\
\hline Sex, male/female & $22 / 6$ \\
Age, median (range), yr & $68(47-81)$ \\
Macroscopic findings & \\
O-IIa & 17 \\
O-IIc & 8 \\
O-IIa+IIc & 3 \\
Location & \\
Upper & 4 \\
Middle & 13 \\
Lower & 11 \\
Histological type & \\
LGD & 8 \\
HGD & 7 \\
WD & 7 \\
MD & 1 \\
PD & 5 \\
\hline
\end{tabular}

IIa, flat elevated; IIc, flat depressed; LGD, low-grade dysplasia; HGD, high-grade dysplasia; WD, well-differentiated adenocarcinoma; MD, moderately differentiated adenocarcinoma; PD, poorly differentiated adenocarcinoma. with poor differentiation in three cases and adenoma in four cases. The mean time to recurrence was 16.1 months (range, 2 to 78 ). The individual time to recurrence was 3 months in three patients and 2, 5, 19, and 78 months in one patient each. With the single APC procedure, three lesions did not recur after more than 64 months. One EGC lesion with poor differentiation recurred after 78 months. The patient had no metastasis and was followed up regularly before the diagnosis of recurrence. The recurring lesion was easily managed by APC retreatment with no complications.

There were no serious APC-related complications such as perforation, bleeding, or infection. Table 2 summarizes the results of 10 patients who were treated with a single APC; Table 3 summarizes the results of 18 patients who were treated with rescue APC following ESD or EMR.

\section{DISCUSSION}

APC has been used to treat a range of gastrointestinal problems including bleeding ulcers, ${ }^{6}$ Dieulafoy's lesions, ${ }^{5}$ hemorrhagic vascular ectasia, ${ }^{7}$ and tumors. ${ }^{8}$ Few reports on APC as a first-line treatment for EGC have been published. The results of clinical trials ${ }^{8-10,12-15}$ of APC for the first-line treatment of EGC or gastric adenoma are summarized in Table 4.

The present study found that APC was useful for the treatment of EGC or gastric adenoma. With a single APC procedure, three lesions did not recur for more than 64 months and one EGC lesion with poor differentiation recurred after 78 months. With the rescue APC procedure, 11\% lesions (2/18) recurred and one EGC lesion has not recurred after more than 57 months.

The recurring lesions were frequently observed to be large. Two local recurrences measured $50 \mathrm{~mm}$. Almost all recurrent lesions were of elevated type IIa rather than the depressed type.

Table 2. Data on Patients Who Underwent Single Argon Plasma Coagulation

\begin{tabular}{|c|c|c|c|c|c|c|c|}
\hline No. & Age/Sex & Tumor size, mm & Macroscopic finding & Location & Pathological type & Duration, $\mathrm{mo}^{\text {a) }}$ & Local recurrence \\
\hline 1 & $77 / \mathrm{M}$ & 30 & $\mathrm{IIa}+\mathrm{c}$ & $\mathrm{L}$ & $\mathrm{PD}$ & 78 & Yes (APC) \\
\hline 2 & $55 / \mathrm{M}$ & 7 & IIa & $\mathrm{L}$ & LGD & 65 & No \\
\hline 3 & $66 / \mathrm{M}$ & 20 & IIa & M & LGD & 3 & Yes (APC) \\
\hline 4 & $74 / \mathrm{M}$ & 25 & IIa & M & LGD & 45 & No \\
\hline 5 & $56 / \mathrm{F}$ & 40 & IIc & M & LGD & 19 & Yes (APC) \\
\hline 6 & $68 / \mathrm{M}$ & 10 & IIa & M & PD & 5 & Yes (ESD) \\
\hline 7 & $81 / \mathrm{M}$ & 20 & IIc & $\mathrm{U}$ & $\mathrm{PD}$ & 2 & Yes (APC) \\
\hline 8 & $68 / \mathrm{M}$ & 10 & IIa & $\mathrm{U}$ & LGD & 72 & No \\
\hline 9 & $68 / \mathrm{F}$ & 10 & IIa & M & HGD & 64 & No \\
\hline 10 & $60 / \mathrm{M}$ & 10 & IIc & $\mathrm{L}$ & HGD & 34 & No \\
\hline
\end{tabular}

M, male; IIa, flat elevated; L, lower third; PD, poorly differentiated adenocarcinoma; APC, argon plasma coagulation; LGD, low-grade dysplasia; M, middle third; F, female; IIc, flat depressed; ESD, endoscopic submucosal dissection; U, upper third; HGD, high-grade dysplasia.

${ }^{\text {a) }}$ Duration, interval between APC procedure and local recurrence. 
Table 3. Data on Patients Who Underwent Rescue Argon Plasma Coagulation

\begin{tabular}{|c|c|c|c|c|c|c|c|}
\hline No. & Age/Sex & Tumor size, $\mathrm{mm}$ & Macroscopic finding & Location & Pathological type & Duration, $\mathrm{mo}^{\mathrm{a})}$ & Local recurrence \\
\hline 1 & $50 / \mathrm{M}$ & 50 & IIa & $\mathrm{U}$ & $\mathrm{HGD} \rightarrow \mathrm{WD}$ & 3 & Yes (surgery) \\
\hline 2 & $51 / \mathrm{F}$ & 20 & IIa & $\mathrm{L}$ & HGD & 50 & No \\
\hline 3 & $67 / \mathrm{M}$ & 40 & IIa & M & $\mathrm{HGD} \rightarrow \mathrm{WD}$ & 45 & No \\
\hline 4 & $65 / \mathrm{M}$ & 20 & $\mathrm{IIa}+\mathrm{c}$ & M & WD & 57 & No \\
\hline 5 & $47 / \mathrm{M}$ & 15 & IIa & M & $\mathrm{HGD} \longrightarrow \mathrm{WD}$ & 21 & No \\
\hline 6 & $66 / \mathrm{M}$ & 30 & IIc & M & HGD & 12 & No \\
\hline 7 & $68 / \mathrm{M}$ & 30 & IIc & $\mathrm{U}$ & $\mathrm{PD}$ & 18 & No \\
\hline 8 & $68 / \mathrm{M}$ & 20 & IIc & M & $\mathrm{WD} \rightarrow \mathrm{MD}$ & 9 & No \\
\hline 9 & $65 / \mathrm{M}$ & 50 & IIa & M & LGD & 3 & Yes (surgery) \\
\hline 10 & $74 / \mathrm{F}$ & 15 & IIa & $\mathrm{L}$ & HGD & 35 & No \\
\hline 11 & $74 / \mathrm{M}$ & 10 & IIa & A & HGD & 5 & No \\
\hline 12 & $70 / \mathrm{M}$ & 5 & IIa & $\mathrm{M}$ & LGD & 3 & No \\
\hline 13 & $72 / \mathrm{M}$ & 20 & IIC & $\mathrm{L}$ & HGD & 7 & No \\
\hline 14 & $66 / \mathrm{M}$ & 20 & $\mathrm{IIa}+\mathrm{c}$ & $\mathrm{L}$ & WD & 8 & No \\
\hline 15 & $62 / \mathrm{M}$ & 10 & IIa & $\mathrm{L}$ & WD & 12 & No \\
\hline 16 & $65 / \mathrm{M}$ & 20 & IIa & $\mathrm{L}$ & LGD & 12 & No \\
\hline 17 & $75 / \mathrm{F}$ & 10 & IIc & $\mathrm{L}$ & $\mathrm{PD}$ & 6 & No \\
\hline 18 & $76 / F$ & 20 & IIa & $\mathrm{L}$ & WD & 3 & No \\
\hline
\end{tabular}

M, male; IIa, flat elevated; U, upper third; HGD, high-grade dysplasia; WD, well-differentiated adenocarcinoma; F, female; L, lower third; M, middle third; IIc, flat depressed; PD, poorly differentiated adenocarcinoma; MD, moderately differentiated adenocarcinoma; LGD, low-grade dysplasia; A, anastomosis site.

a) Duration, interval between APC procedure and local recurrence.

Table 4. Clinical Trials of Argon Plasma Coagulation for the First-Line Treatment of Early Gastric Cancer or Gastric Adenoma

\begin{tabular}{|c|c|c|c|c|}
\hline Study & Patient characteristic, lesion & $\begin{array}{l}\text { Duration, median } \\
\quad \text { (range), mo }\end{array}$ & $\begin{array}{c}\text { Local recurrence, } \\
\text { lesion }(\%)^{\mathrm{b})}\end{array}$ & $\begin{array}{l}\text { Recurrence } \\
\text { interval, mo }\end{array}$ \\
\hline Sagawa et al. $(2003)^{8}$ & 27 with EGC & $30(18-49)$ & $1(3.7)$ & 6 \\
\hline Tomita et al. $(2011)^{9}$ & 50 (25 EGC, 25 adenoma) & $43.2(6-79.2)$ & $5(10)$ & 18 \\
\hline Ahn et al. $(2013)^{10}$ & 71 (10 EGC, 61 adenoma) & $20(13-29)$ & $15(21)$ & $10(5-13)$ \\
\hline Murakami et al. $(2004)^{12}$ & 23 with EGC & 42 & $4(17)$ & - \\
\hline Kitamura et al. $(2006)^{13}$ & 40 with EGC & $52.4(2.5-71.9)$ & $4(10)$ & $3.2(1.3-4.7)$ \\
\hline Lee et al. $(2009)^{14}$ & 64 with adenoma (LGD) & $19.5(6-49)$ & $4(6.3)$ & $5.8(3-9)$ \\
\hline \multirow[t]{2}{*}{ Jung et al. $(2013)^{15}$} & 320 with adenoma (LGD) (116 APC, 204 ESD) & $22.5(2.2-64.4)(\mathrm{APC})$ & $4(\mathrm{APC})$ & $2.8-14.6(\mathrm{APC})$ \\
\hline & & $37.1(1-88.2)(\mathrm{ESD})$ & 1 (ESD) & 4.7 (ESD) \\
\hline Present study & 28 (13 EGC, 15 adenoma) & $24.8(2-78)$ & $7(25)$ & $16.1(2-78)$ \\
\hline
\end{tabular}

EGC, early gastric cancer; LGD, low-grade dysplasia; APC, argon plasma coagulation; ESD, endoscopic submucosal dissection.

${ }^{a)}$ Duration, follow-up period from the date of initial APC treatment to the date of final endoscopic examination or recurrence; ${ }^{\text {b) }}$ Recurrence interval, period from the date of initial APC treatment to the date of local recurrence.

This is potentially due to insufficient coagulation by APC therapy, since the tumor volume is greater in type IIa lesions than in depressed type lesions of the same size. APC is therefore a satisfactory and effective modality for flat and depressed lesions of a relatively small size. This finding has been notedin other studies. ${ }^{9,13,15}$ One study observed that all recurrent lesions were of elevated type IIa, regardless of the endoscopist's experience. ${ }^{9}$ Another study found that all recurrent lesions follow- ing APC were of the superficial elevated type. ${ }^{15}$ Yet another study found that no patient with intramucosal carcinoma had a residual tumor or recurrence after a single session or multiple sessions of APC. ${ }^{13}$ By contrast, three of the five patients (60\%) with submucosal tumors had a residual tumor or recurrence. One of these three patients had large tumors that were treated with a single session of APC. The remaining two patients received two sessions of APC and had no evidence of local re- 
currence. This suggests that large protruding-type tumors or tumors with submucosal invasion have a high risk of residual tumor cells because of inadequate treatment after one session of APC..$^{13}$

In a study in which 27 patients received APC for the treatment of EGC, 26 showed no recurrence during 30 months of follow-up. ${ }^{8}$ Most studies have shown that APC is an effective and safe treatment option for early gastric neoplasms (Table 4). APC is useful for the follow-up treatment of EGC after EMR. ${ }^{12}$ Taken together, these results suggest that the APC procedure canbe a therapeutic strategy for EGC, especially in elderly and high-risk patients. However, the risk factors for local recurrence have not been adequately studied. Non-lifting after submucosal saline injection and a lower power setting of $40 \mathrm{~W}$ are both risk factors for local recurrence after APC, with a lower reported rate of local recurrence in lifting and 60 or $80 \mathrm{~W}$ groups than in non-lifting and $40 \mathrm{~W}$ groups. ${ }^{10} \mathrm{In}$ that study, the authors concluded that a higher power setting of 60 or $80 \mathrm{~W}$ may be safe and effective for the treatment of gastric neoplasms by APC. ${ }^{10}$

In endoscopic treatment, histological assessment is crucial for determining whether the lesions were treated successfully and whether additional surgery is needed. However, histology cannot be evaluated in APC treatment because the coagulation resulting from APC causes necrosis of the tumorous tissue. This is one reason that APC treatment alone has not been used as a first-line therapy for EGC until now. However, a strict histological assessment of the resected specimen may not always be necessary in patients without operable justification. The treatment options for EGC are nonuniform, even if tumors can be treated by endoscopic resection. Consequently, a different approach to the treatment of EGC may be selected in primary or geriatric hospitals. ${ }^{9}$

The treatment of low-grade dysplasia (LGD) can be different from that of high-grade dysplasia or EGC. APC has been shown to be a suitable treatment option for patients with a gastric LGD $\leq 2.0 \mathrm{~cm}$ when compared with ESD. ${ }^{15}$ Because of reduced hospital admissions, lower medical costs, fewer complications, and more favorable outcomes, APC can be considered a first-line treatment option for patients with small gastric LGDs. Moreover, a recent study ${ }^{9}$ showed that the outcomes of APC did not differ according to endoscopic experience, with equal therapeutic outcomes achieved by experienced and nonexperienced endoscopists. Therefore, non-experienced endoscopists may treat small gastric LGD lesions without a long training period such as that required for ESD., ${ }^{9,15}$

Our study had several limitations. First, this was a singlecenter study with a limited number of patients. It was a retrospective cohort study that involved the review of the records of patients with EGCs or adenomas. Second, there was no con- trol group. The relatively short duration of follow-up may be another limitation. Further randomized prospective studies are necessary to confirm that APC is an effective and reasonable treatment for early gastric neoplasms.

In conclusion, APC can be a favorable therapeutic modality for the rescue treatment of early gastric neoplasms after EMR or ESD. In addition, APC can bea safe and effective first-line treatment of early gastric neoplasms, especially in elderly and high-risk patients.

\section{Conflicts of Interest}

The authors have no financial conflicts of interest.

\section{REFERENCES}

1. Gotoda T, Yamamoto H, Soetikno RM. Endoscopic submucosal dissection of early gastric cancer. J Gastroenterol 2006;41:929-942.

2. Ono H, Kondo H, Gotoda T, et al. Endoscopic mucosal resection for treatment of early gastric cancer. Gut 2001;48:225-229.

3. Ahn JY, Jung HY, Choi KD, et al. Endoscopic and oncologic outcomes after endoscopic resection for early gastric cancer: 1370 cases of absolute and extended indications. Gastrointest Endosc 2011;74:485-493.

4. Grund KE, Storek D, Farin G. Endoscopic argon plasma coagulation (APC) first clinical experiences in flexible endoscopy. Endosc Surg Allied Technol 1994;2:42-46.

5. Wahab PJ, Mulder CJ, den Hartog G, Thies JE. Argon plasma coagulation in flexible gastrointestinal endoscopy: pilot experiences. Endoscopy 1997;29:176-181.

6. Chau CH, Siu WT, Law BK, et al. Randomized controlled trial comparing epinephrine injection plus heat probe coagulation versus epinephrine injection plus argon plasma coagulation for bleeding peptic ulcers. Gastrointest Endosc 2003;57:455-461.

7. Sebastian S, McLoughlin R, Qasim A, O’Morain CA, Buckley MJ. Endoscopic argon plasma coagulation for the treatment of gastric antral vascular ectasia (watermelon stomach): long-term results. Dig Liver Dis 2004; 36:212-217.

8. Sagawa T, Takayama T, Oku T, et al. Argon plasma coagulation for successful treatment of early gastric cancer with intramucosal invasion. Gut 2003;52:334-339.

9. Tomita T, Arai E, Kohno T, et al. Outcomes of treatment of argon plasma coagulation therapy in elderly or high-risk patients with early gastric cancer: a comparison of outcomes among experienced and nonexperienced endoscopists. J Clin Gastroenterol 2011;45:e54-e59.

10. Ahn JY, Choi KD, Na HK, et al. Clinical outcomes of argon plasma coagulation for the treatment of gastric neoplasm. Surg Endosc 2013;27: 3146-3152.

11. Japanese Gastric Cancer Association. Japanese classification of gastric carcinoma: 2nd English edition. Gastric Cancer 1998;1:10-24.

12. Murakami M, Nishino K, Inoue A, et al. Argon plasma coagulation for the treatment of early gastric cancer. Hepatogastroenterology 2004;51: 1658-1661.

13. Kitamura T, Tanabe S, Koizumi W, Mitomi H, Saigenji K. Argon plasma coagulation for early gastric cancer: technique and outcome. Gastrointest Endosc 2006;63:48-54.

14. Lee KM, Kim YB, Sin SJ, et al. Argon plasma coagulation with submucosal saline injection for gastric adenoma on outpatient basis. Dig Dis Sci 2009;54:2623-2628.

15. Jung SJ, Cho SJ, Choi IJ, et al. Argon plasma coagulation is safe and effective for treating smaller gastric lesions with low-grade dysplasia: a comparison with endoscopic submucosal dissection. Surg Endosc 2013; 27:1211-1218. 